\title{
Curcumin inhibits autophagy and apoptosis in hypoxia/reoxygenation-induced myocytes
}

\author{
ZHOUQING HUANG ${ }^{1}$, BOZHI YE ${ }^{1}$, ZHENYU DAI $^{1}$, XINLEI WU $^{1}$, \\ ZHONGQIU LU ${ }^{2}$, PEIREN SHAN ${ }^{1}$ and WEIJIAN HUANG ${ }^{1}$ \\ Departments of ${ }^{1}$ Cardiology and ${ }^{2}$ Emergency, The Key Laboratory of Cardiovascular Disease of Wenzhou, \\ The First Affiliated Hospital of Wenzhou Medical University, Wenzhou, Zhejiang 325000, P.R. China
}

Received May 2, 2014; Accepted December 2, 2014

DOI: $10.3892 / \mathrm{mmr} .2015 .3322$

\begin{abstract}
Primary percutaneous coronary intervention, or thombolytic therapy, provides effective myocardial blood reconstruction in patients with acute myocardial infarction to reduce acute myocardial ischemic injury. However, reperfusion can itself induce cardiomyocyte death, termed myocardial reperfusion injury (I/R). Hypoxia/reoxygenation $(\mathrm{H} / \mathrm{R})$ induces apoptosis and excessive autophagy among cardiomyocytes, leading to cell death. The present study investigated the effect of curcumin, a natural extract from Curcuma longa, on these two cellular processes in H9c2 myocytes. The levels of cellular apoptosis and autophagy were found to be upregulated in the $\mathrm{H} 9 \mathrm{c} 2$ myocytes during $\mathrm{H} / \mathrm{R}$ and were correlated with a reduced rate of cell survival. However, curcumin significantly suppressed the levels of $\mathrm{H} / \mathrm{R}$-induced apoptosis (expression of annexin V) and autophagy (LC3B-II/LC3B-I ratio) in the $\mathrm{H} 9 \mathrm{c} 2$ myocytes and promoted cell survival. Additionally, the expression of B-cell lymphoma 2 (Bcl-2) was significantly downregulated and the expression levels of Bcl-2-associated X protein, beclin-1, Bcl-2/adenovirus E1B $19 \mathrm{kDa}$ interacting protein 3 (BNIP3) and silent information regulation 1 (SIRT1) were significantly upregulated in myocytes following H/R injury. These effects on the expression of these proteins were reversed by curcumin treatment. These findings suggested that the protective effect of curcumin against H/R injury in the H9c2 myocytes was through the inhibition of apoptosis and autophagy by inducing the expression of Bcl-2 and inhibiting the expression levels of Bax, beclin-1, BNIP3 and SIRT1. Therefore, curcumin may offer a promising therapeutic approach for the treatment of cardiomyocyte injury resulting from $\mathrm{I} / \mathrm{R}$.
\end{abstract}

Correspondence to: Professor Weijian Huang, Department of Cardiology, The Key Laboratory of Cardiovascular Disease of Wenzhou, The First Affiliated Hospital of Wenzhou Medical University, 2 Fuxue Road, Wenzhou, Zhejiang 325000, P.R. China E-mail: weijianhuang69@126.com

Key words: curcumin, autophagy, apoptosis, hypoxia/reoxygenation injury, myocytes

\section{Introduction}

Acute myocardial infarction (MI) is a major cause of death worldwide (1). Despite the availability of primary percutaneous coronary intervention or thrombolytic therapy as the most effective treatment for myocardial blood reconstruction in patients who are suffering from MI (2), myocardial ischemia/reperfusion (I/R) injury remains the major cause of heart failure worldwide (3). This type of injury activates apoptosis and autophagy, which results in cell death (4). Therefore, an increasing number of studies have investigated the roles of apoptosis and autophagy during I/R injury in myocytes (5-7).

Apoptosis is an active energy-dependent mode of cell death, which is regulated by programmed cellular signaling pathways. Previous studies have demonstrated that I/R-induced myocyte injury results in apoptosis and the suppression of cardiomyocyte apoptosis provides significant cardioprotection against I/R injury (8-10). By contrast, autophagy is a natural biological process, which removes unnecessary proteins and damaged organelles and maintains cellular homeostasis (11). The upregulation of autophagy during mild ischemia is associated with the preservation of the mitochondrial membrane potential and cellular membrane integrity, accompanied by a significant delay in the onset of apoptosis and necrosis (12). However, excessive autophagy damages essential proteins and organelles, leading to collapse of all cellular functions (13). Evidence supporting this concept by Matsui et al demonstrated that excessive autophagy may be detrimental to the heart during reperfusion (14). Therefore, the identification of treatments with anti-apoptotic and anti-autophagic effects is important for protection of the heart from I/R injury. The investigation of traditional medicines as a potential treatment option in I/R injury has received interest due to their wide range of pharmacological effects (15-16).

Curcumin, a natural polyphenolic compound present in the rhizomes of Curcuma longa, has potential anti-inflammatory, antioxidant, anti-carcinogenic and cardiovascular protective effects (17-19). Previous studies have demonstrated that curcumin attenuates $\mathrm{I} / \mathrm{R}$ injury in various organs in vivo and in vitro, including the intestines, retina, liver and renal system, through anti-oxidative stress and anti-apoptotic mechanisms (20-23). In addition, Gonzalez-Salazar et al (24) demonstrated that curcumin mitigated myocardial I/R injury 
through the attenuation of oxidative stress and mitochondrial dysfunction. However, the beneficial effects of curcumin on the extent of apoptosis and autophagy in myocardial I/R, and their potential mechanisms, remain to be elucidated.

The present study examined the protective effect of curcumin on hypoxia/reoxygenation (H/R)-induced $\mathrm{H} 9 \mathrm{c} 2$ myocytes, focusing on the regulation of apoptosis and autophagy. The potential mechanism by which curcumin mediates these processes was also investigated.

\section{Materials and methods}

Reagents. Dulbecco's modified Eagle's medium (DMEM), fetal bovine serum (FBS) and 10,000 U/ml penicillin/streptomycin were obtained from Gibco Life Technologies (Carlsbad, CA, USA). Dimethyl sulfoxide (DMSO) and curcumin were obtained from Sigma-Aldrich (St. Louis, MO, USA). Rabbit monoclonal antibody against GAPDH was produced by Abcam (Cambridge, UK). Rabbit monoclonal anti-LC3B antibody was provided by Zymed Life Technologies (San Francisco, CA, USA). Rabbit monoclonal anti-beclin-1, Bcl-2/adenovirus E1B $19 \mathrm{kDa}$ interacting protein 3 (BNIP3) and silent information regulation 1 (SIRT1) antibodies were purchased from the Cell Signaling Technology, Inc. (Danvers, MA, USA). Goat anti-rabbit secondary antibody (cat. no. A-21109) was purchased from Invitrogen Life Technologies (Carlsbad, CA, USA). A cellular adenosine triphosphate (ATP) kit was obtained from Beyotime Institute of Biotechnology (Shanghai, China). All other chemicals for western blot analysis were of the highest commercial purity grade available.

Cell culture and treatment. The $\mathrm{H} 9 \mathrm{c} 2$ rat myocardium-derived cardiac myoblast cell line was purchased from the American Type Culture Collection (Rockville, MD, USA). The cells were cultured in DMEM containing 4,500 mg/l glucose and supplemented with $10 \%$ (v/v) FBS, $10 \mathrm{mM}$ HEPES (Sigma-Aldrich) and $1 \%$ penicillin/streptomycin at $37^{\circ} \mathrm{C}$ in a $5 \% \mathrm{CO}_{2}$ incubator for $24 \mathrm{~h}$. Prior to pretreatment with curcumin, the cell media were replaced with FBS-free media for $4 \mathrm{~h}$ for cell cycle synchronization until the cell density conformed with the experimental standard of achieving a unified cell state. To identify the cytotoxicity of curcumin on $\mathrm{H} 9 \mathrm{c} 2$ myocytes, the cells $\left(1 \times 10^{6} / \mathrm{ml}\right)$ were pretreated with curcumin $(1,5,10,20,40$ and $80 \mu \mathrm{M})$ for $1 \mathrm{~h}$ under normoxic conditions with an atmosphere of $21 \% \mathrm{O}_{2}, 5 \% \mathrm{CO}_{2}$ and $74 \% \mathrm{~N}_{2}$ in FBS- and glucose-free DMEM. To simulate starvation, the cells were cultured in FBS- and glucose-free DMEM, with the exception of the normal control group cells which were cultured in complete DMEM. To simulate I/R injury, the glucose-deprived cells were cultured under hypoxic conditions with an atmosphere of $1 \% \mathrm{O}_{2}, 5 \% \mathrm{CO}_{2}$ and $94 \% \mathrm{~N}_{2}$ for $1 \mathrm{~h}$ and then cultured under normoxic conditions for $3 \mathrm{~h}$. The cells in the normal control and starvation groups were exposed to normoxic conditions for $4 \mathrm{~h}$.

Cell viability detection by 3-(4,5-dimethylthiazol-2-yl)-2,5-diphenyltetrazolium bromide (MTT) assay. An MTT assay was performed to assess the cell viability following treatment with curcumin $(1,5,10,20,40$ and $80 \mu \mathrm{M})$. Subsequent to the previously described treatments, the cells were incubated with
$0.5 \mathrm{mg} / \mathrm{ml}$ MTT (Roche Diagnostics, Basel, Switzerland) in RPMI-1640 medium (Life Technologies, Carlsbad, CA, USA) for a further $4 \mathrm{~h}$. The blue formazan crystals of the viable cells were dissolved in $150 \mu \mathrm{l}$ DMSO and the absorbance was measured at a wavelength of $570 \mathrm{~nm}$ using a spectrophotometer (DNM-9602; Perlong, Beijing, China).

ATP determination. The intracellular levels of ATP were determined using an ATP bioluminescent assay kit (Beyotime Institute of Biotechnology) according to the manufacturer's instructions.

Annexin V/propidium iodide (PI) double staining and flow cytometry. The apoptotic rate was measured by flow cytometry, which was performed using primary mouse anti-human monoclonal antibodies and fluorescein isothiocyanate (FITC)/PI double staining-annexin V (BD Biosciences, Franklin Lakes, NJ, USA) according to the manufacturer's instructions. The cells were incubated with $5 \mu \mathrm{l}$ annexin V-FITC and PI at room temperature for $15 \mathrm{~min}$ in the dark and apoptosis was detected by flow cytometry (BD accuri C6; BD Biosciences). The mean fluorescent intensity of the annexin V/PI double staining in the myocytes was analyzed using a BD fluorescent activated cell sorter (FACS) calibur (BD Biosciences).

Protein isolation and western blot analysis. Protein isolation and western blot analyses were performed, as previously described (25), with the exception that the membranes were probed with either rabbit primary Bcl-2-associated $\mathrm{X}$ protein (Bax), Bcl-2, LC3B-II/I, beclin-1, BNIP3 or SIRT1 antibodies diluted 1:1,000 in Tris-buffered saline containing Tween-20 (TBST; Solarbio, Beijing, China) for $2 \mathrm{~h}$ or rabbit anti-GAPDH diluted 1:5,000 in TBST for $1 \mathrm{~h}$. The membranes were then incubated with goat anti-rabbit secondary antibody labeled with far-red-fluorescent AlexaFluor 680 dye (1:1,000 in TBST; Abcam, Cambridge, UK). All signals were detected using the Odyssey CLx Infrared Imaging system (Li-Cor Biosciences, Lincoln, NE, USA). Densitometric analysis was performed using the Quantity One system (170-9600; Bio-Rad Laboratories, Inc., Hercules, CA, USA).

Statistical analysis. Groups of three or more were compared using one-way analysis of variance (ANOVA) with Student-Newman-Keuls and Dunnet methods as post-hoc analysis if the result of the ANOVA was significant. The data are expressed as the mean \pm standard deviation and were analyzed using SPSS 18.0 software (SPSS, Inc., Chicago, IL, USA). $\mathrm{P}<0.05$ was considered to indicate a statistically significant difference. All experiments were performed at least three times.

\section{Results}

Effect of curcumin on cell viability. An MTT assay was used to assess the effect of curcumin on the viability of the H9c2 myocytes. As shown in Fig. 1 the concentrations of curcumin, ranging between 1 and $20 \mu \mathrm{M}$, had no significant effect on cell viability. Therefore, a curcumin concentration $<20 \mu \mathrm{M}$ was considered non-cytotoxic and a concentration of $10 \mu \mathrm{M}$ was used in the subsequent experiments. As shown 


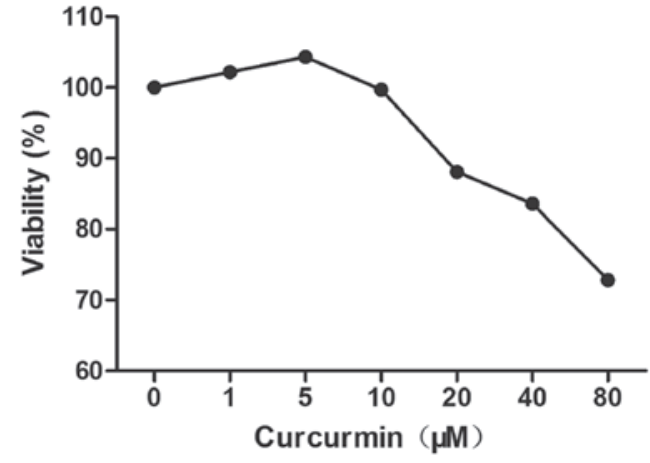

Figure 1. Effect of curcumin treatment on the viability of the H9c2 myocytes in glucose-free Dulbecco's modified Eagle's medium. The cells were treated with various concentrations of curcumin $(1,5,10,20,40$ and $80 \mu \mathrm{M})$ for $24 \mathrm{~h}$ and the cell viability was assessed using a 3-(4,5-dimethylthiazol-2-yl)-2,5-diphenyltetrazolium bromide assay. Cells incubated in curcumin-free medium were used as a control and were considered $100 \%$ viable.

A

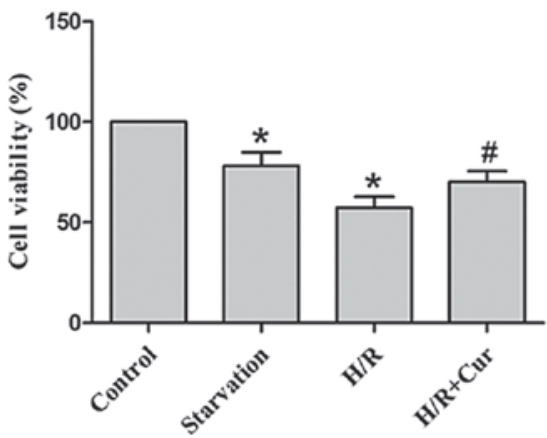

B

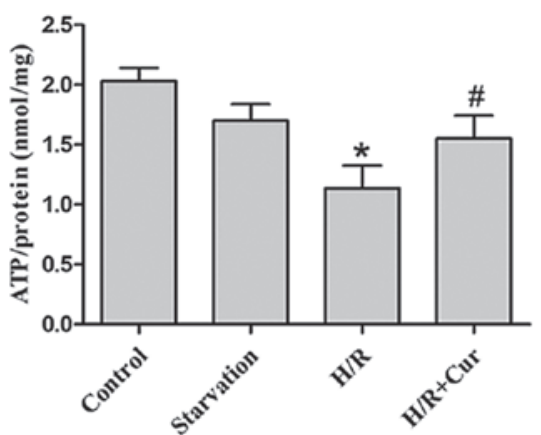

Figure 2. Effect of curcumin on the viability of the H9c2 cells and the cellular ATP content during H/R. (A) Cells were treated with $10 \mu \mathrm{M}$ curcumin and cell viability was assessed after $24 \mathrm{~h}$ using a 3-(4,5-dimethylthiazol-2-yl)-2,5-diphenyltetrazolium bromide assay. The cells incubated in complete curcumin-free Dulbecco's modified Eagle's medium were used as a control group and considered as $100 \%$ viable. (B) Curcumin reversed the depletion of cellular ATP in the H9c2 cells subjected to H/R. Data are expressed as the mean \pm standard deviation of three independent experiments ( $\mathrm{P}<0.01$ compared with the control group; ${ }^{*} \mathrm{P}<0.05$ compared with the $\mathrm{H} / \mathrm{R}$ group). H/R, hypoxia/reoxygenation; Cur, curcumin; ATP, adenosine triphosphate.

in Fig. 2A, the viability of the H9c2 cells was significantly reduced in the starvation and $\mathrm{H} / \mathrm{R}$ treatment groups. Treatment with $10 \mu \mathrm{M}$ curcumin increased the viability of the H9c2 cells during the H/R period compared with the control group.

Curcumin abrogates the depletion of cellular ATP in H9c2 cells exposed to $H / R$. The starvation and $\mathrm{H} / \mathrm{R}$ treatments resulted in the depletion of cellular ATP contents in the H9c2 myocytes. Treatment with curcumin significantly reversed the depletion of cellular ATP, which improved cell survival (Fig. 2B).

Curcumin inhibits apoptosis in the H9c2 myocytes. The present study aimed to determine whether apoptosis was involved in the effect of curcumin on cell viability. Annexin V/PI double staining was used to assess the apoptotic rate in the H9c2 cells. The results demonstrated that the expression of annexin $\mathrm{V}$ was significantly increased in the starvation (glucose deprivation; GD) and $\mathrm{H} / \mathrm{R}$ groups compared with the normal group. However, an apparent reduction in the expression of annexin $\mathrm{V}$ was observed in the H/R-induced H9c2 myocytes, which were pretreated with curcumin (Fig. 3A).

The $\mathrm{Bcl}-2$ protein family is important in the regulation of apoptosis. The anti-apoptotic protein, Bcl-2, is mainly located in the mitochondria, protecting these organelles by preventing the movement of pro-apoptotic proteins, including Bax, Bcl-2-antagonist/killer 1 and Bcl-2 homology 3 (BH3) interacting-domain, to the mitochondria (26). Therefore, the expression levels of these proteins were assessed to determine the initial apoptotic events caused by curcumin. As shown in Fig. 3B, H/R activated the expression of Bax and downregulated the expression of $\mathrm{Bcl}-2$ in the $\mathrm{H} 9 \mathrm{c} 2$ myocytes. Treatment with curcumin markedly reversed these effects on the expression levels of Bcl-2 and Bax in the cells exposed to H/R.

Curcumin inhibits autophagy in the H9c2 myocytes. In mammalian cells, LC3B-II is produced from LC3B-I and is modified into a membrane-bound form to prompt its localization to autophagosomes. Thus, LC3B-II is considered to be an autophagosomal marker (27). The present study investigated the expression of LC3B-II using western blot analysis. As shown in Fig. 4A and B, the ratio of LC3B-II/LC3B-I was increased in response to $H / R$ and reduced by curcumin in the H9c2 myocytes.

Autophagy is a complex biological process involving several distinct regulatory modules. Therefore, the present study further examined autophagy-associated proteins, including beclin-1, SIRT1 and BNIP3, to confirm whether they were involved in the effects of curcumin on the level of autophagy in myocardial $\mathrm{H} / \mathrm{R}$ injury. The results demonstrated that the expression levels of beclin-1, SIRT1 and BNIP3 were markedly upregulated in the GD and $\mathrm{H} / \mathrm{R}$ groups and were significantly downregulated by treatment with curcumin (Fig. 4C and D). These results suggested that curcumin affected the level of autophagy by downregulating the expression levels of beclin-1, SIRT1 and BNIP3 in the H9c2 myocytes during H/R injury.

\section{Discussion}

In present study, curcumin significantly increased the viability of the H9c2 myocytes subjected to H/R by inhibiting the levels of apoptosis and autophagy. Upregulation of the expression of Bcl-2 and downregulation of the expression of Bax were involved in the inhibitory effect of curcumin on the apoptosis of myocytes during H/R. Additionally, suppression of the expression levels of beclin-1, SIRT1 and BNIP3 were identified as necessary molecular mechanism by which curcumin mitigated autophagy activity during myocardial H/R injury. 
A
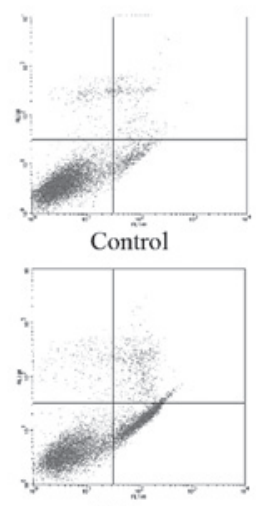

$\mathrm{H} / \mathrm{R}$

C
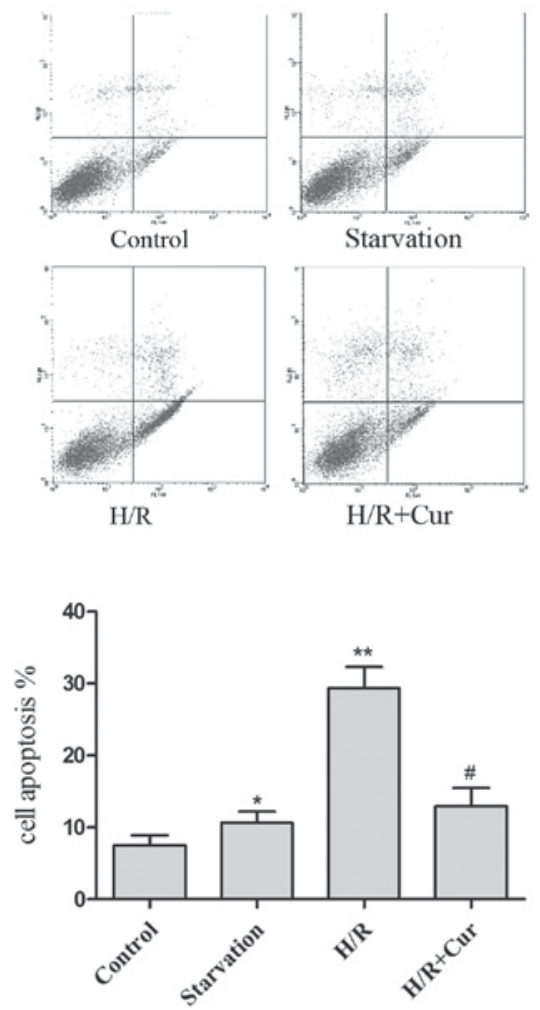

$\mathrm{H} / \mathrm{R}+\mathrm{Cur}$
B

D

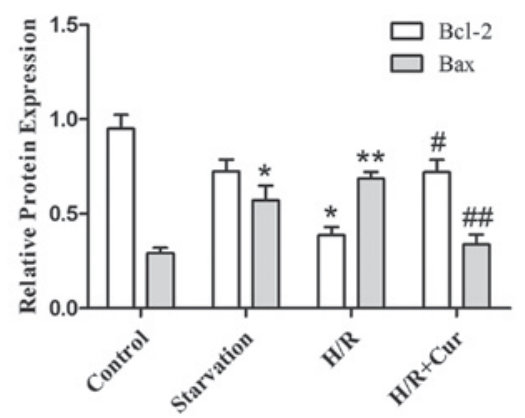

Figure 3. Curcumin inhibits apoptosis in the H9c2 myocytes exposed to H/R. (A) Cells were stained using annexin V (0.5 mg/ml) and propidium iodide $(2 \mathrm{mg} / \mathrm{ml})$ following curcumin treatment for FACS analysis. (B) Representative western blot analysis. (C) Quantitative data from FACS analysis demonstrated that curcumin inhibited apoptosis in the H9c2 myocytes exposed to H/R. (D) Densitometric measurement of Bax and Bcl-2. The band densities were measured using the Quantity One 1D analysis software program. Data are expressed as the mean \pm standard deviation and all experiments were conducted in triplicate and the results were analyzed for statistical significance ${ }^{*} \mathrm{P}<0.05$ and ${ }^{* *} \mathrm{P}<0.01$, compared with the control group; ${ }^{*} \mathrm{P}<0.01$ and ${ }^{\# \prime} \mathrm{P}<0.01$, compared with the $\mathrm{H} / \mathrm{R}$ group). H/R, hypoxia/reoxygenation; Cur, curcumin; Bcl-2, B-cell lymphoma 2; Bax, Bcl-2-associated X protein.

Apoptosis is a well-characterized, specific morphological aspect of cell death, accompanied with nuclear pyknosis (chromatin condensation) and karyorhexis (nuclear fragmentation) (28) and is linked with I/R-induced myocardial injury. The Bcl-2 family protein, Bax, is a pro-apoptotic protein, which forms complexes with other proteins in the mitochondrial outer membrane, promoting the release of pro-apoptotic molecules, including cytochrome $\mathrm{C}$, and activates apoptosis (29). Bcl-2 is an anti-apoptotic molecule that reduces myocardial apoptosis during reperfusion (30). Bcl-2 inhibits the action of Bax through direct or indirect mechanisms and reduces cell death $(31,32)$. The present study demonstrated that the expression of $\mathrm{Bcl}-2$ was upregulated and the expression of Bax was downregulated in the $\mathrm{H} 9 \mathrm{c} 2$ myocytes treated with curcumin, promoting cell survival during H/R. Therefore, curcumin inhibited apoptosis by affecting the expression levels of Bax and Bcl-2.

Autophagy has a dual role in cell survival and cell death during heart ischemia and reperfusion (33). Induction of autophagy maintains the homeostasis of cellular ATP and promotes cell survival in the ischemic heart (34). However, excessive autophagy is detrimental and can lead to myocyte death during the reperfusion phase (14). The present study established a model of $\mathrm{H} / \mathrm{R}$ damage in $\mathrm{H} 9 \mathrm{c} 2$ myocytes to investigate the effect of curcumin on the level of autophagy. The ratio of LCB3-II/LC3B-I increased significantly and was associated with a reduction in cell viability in the myocyte $H / R$ period, suggesting that autophagy during the reperfusion phase may be detrimental for myocyte survival. Notably, curcumin treatment significantly downregulated the ratio of LC3B-II/ LC3B-I and improved the survival rate of the H9c2 myocytes. These results indicated that curcumin promoted cell survival through inhibiting the excessive activation of autophagy by $\mathrm{H} / \mathrm{R}$.

Several molecular mechanisms, including beclin-1, BNIP3 and SIRT1, respond to the regulation of autophagy (35-37). Therefore, the present study examined the changes in the levels of these autophagy-associated proteins in myocytes during H/R. Beclin-1, a BH3 domain-only protein (38), is necessary for activating autophagy during reperfusion and exacerbates cardiomyocyte death (39). Downregulation of the expression of beclin-1 using RNA interference in myocytes reduces the level of I/R-induced autophagy, accompanied by an increased cell survival (40). These results indicated that inhibiting the expression of beclin-1 is beneficial for the survival of myocytes. It has been previously demonstrated that the upregulation of $\mathrm{Bcl}-2$ is important in suppressing autophagy $(26,41,42)$. Bcl-2 binds to beclin-1 via the BH3 domain to form a Bcl-2:beclin-1 complex. This complex prevents beclin-1 from assembling the pre-autophagosomal structure, thereby inhibiting autophagy (43). Notably, a previous study revealed that suppressing the expression of Bcl-2 using small interfering RNA in breast cancer cells did not activate apoptosis as expected, but induced autophagic cell death accompanied by an upregulation in the expression of beclin-1 (44). Due to the association between Bcl-2 and 

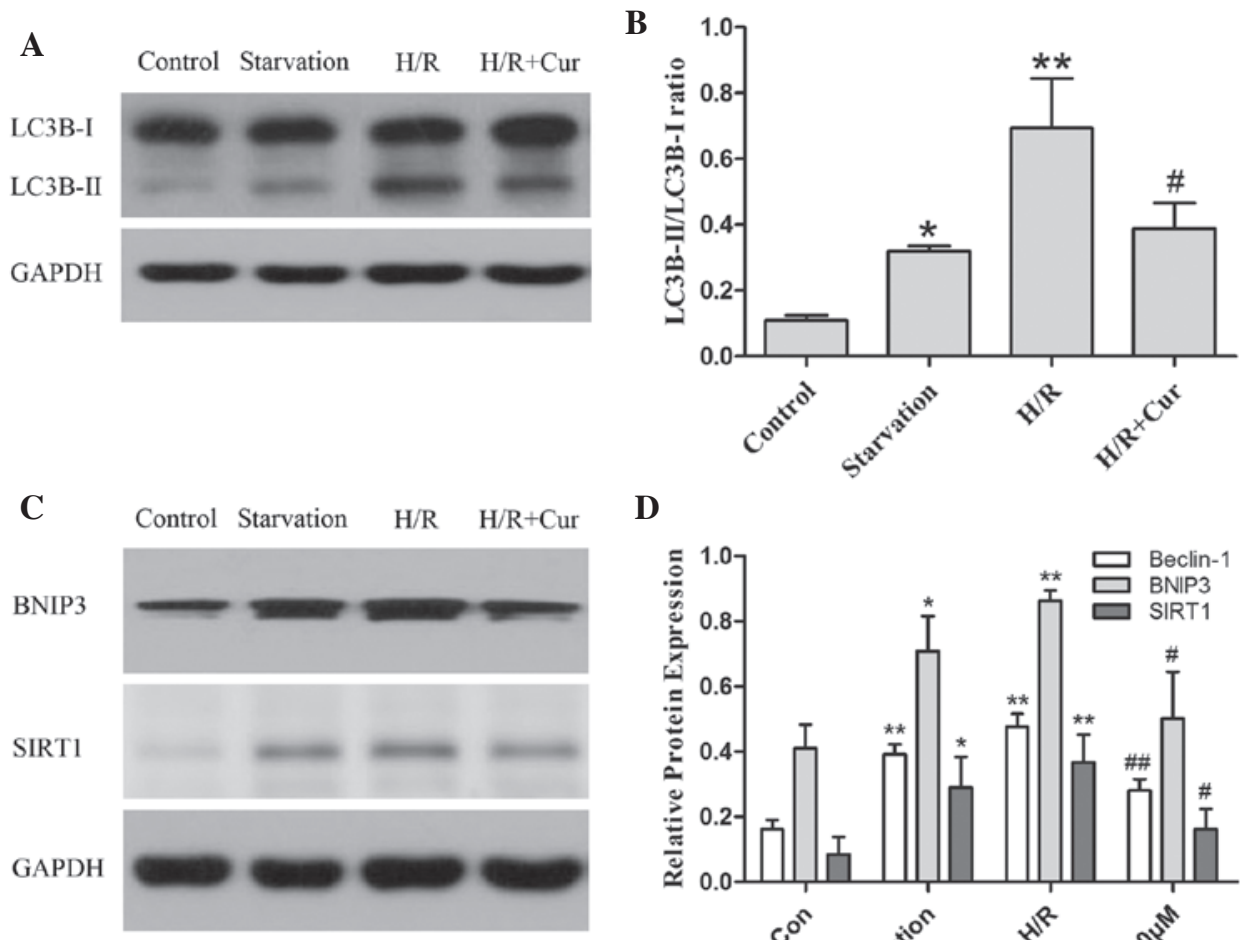

D

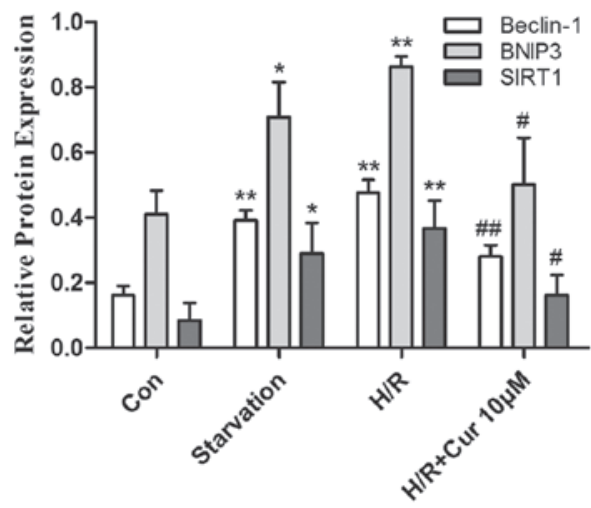

Figure 4. Curcumin downregulates the ratio of LC3B-II/LC3B-I, a marker of autophagy and autophagy-associated proteins, including beclin-1, BNIP3 and SIRT1 in H9c2 myocytes during H/R. The cells were subjected to glucose deprivation, with the exception of the control group, and were cultured under hypoxic conditions for $1 \mathrm{~h}$ and then reoxygenated for $3 \mathrm{~h}$, with or without curcumin. The cell lysates from the H9c2 cells were subjected to (A) western blot analysis. and (B) densitometric measurements of LC3B-II/LC3B-I. (C) Representative western blot analysis and (D) densitometric measurements of beclin-1, BINP3, and SIRT1. The band densities were measured using the Quantity One 1D analysis software program. Data are expressed as the mean \pm standard deviation and were obtained from three independent experiments ( ${ }^{*} \mathrm{P}<0.05$ and ${ }^{* * *} \mathrm{P}<0.01$, compared with the control group; ${ }^{\#} \mathrm{P}<0.05$ and ${ }^{\# \#} \mathrm{P}<0.01$, compared with the $\mathrm{H} / \mathrm{R}$ group). H/R, hypoxia/reoxygenation; Cur, curcumin; BNIP3, adenovirus E1B $19 \mathrm{kDa}$ interacting protein 3 SIRT1, silent information regulation 1.

beclin-1 and the importance of the Bcl-2/beclin-1 complex for autophagy, the present study examined the expression levels of these proteins in myocytes during $H / R$. The results demonstrated that the expression of Bcl-2 was downregulated and the expression of beclin-1 was upregulated, accompanied by an upregulation in autophagy in the myocytes during $\mathrm{H} / \mathrm{R}$. However, these changes in the expression levels of Bcl-2 and beclin-1 and the ratio of LC3B-II/LC3B-I were markedly reversed by curcumin treatment. Therefore, it was suggested that curcumin may upregulate the quantity of the Bcl-2/ beclin-1 complex and downregulate the expression of beclin-1, thereby inhibiting autophagy in the H9c2 myocytes during H/R.

BNIP3, another member of the BH3-contaning Bcl-2 family, is responsible for excessive autophagy and the overexpression of BNIP3 significantly induces autophagy (45). Bellot et al (46) demonstrated that hypoxia-induced BNIP3 induces autophagy by disrupting the Bcl-2/beclin-1 complex. Additionally, overexpression of BNIP3 promoted apoptotic cell death $(47,48)$. SIRT1, a nicotinamide adenine dinucleotide-dependent deacetylase, regulates critical metabolic and physiological processes, including autophagy (37). SIRT1 induces autophagy by forming a molecular complex with several autophagy-associated genes and directly deacetylating these components (49). The overexpression of SIRT1 is sufficient to induce an autophagic flux (50), and SIRT1-/- mouse embryonic fibroblasts fail to fully activate autophagy under conditions of starvation (49). The upregulation of BNIP3 and SIRT1 stimulates autophagy or apoptosis in different cell types $(51,52)$. In the present study the expression levels of BNIP3 and SIRT1 were significantly upregulated during $\mathrm{H} / \mathrm{R}$ in myocytes and curcumin treatment significantly downregulated the expression levels of BNIP3 and SIRT1. This was accompanied by downregulation in the levels of apoptosis and autophagy in the H9c2 myocytes during H/R. Therefore, curcumin inhibited cell apoptosis/excessive autophagy and promoted cell survival by downregulating BNIP3 and SIRT1. Therefore, the results suggested that curcumin assisted in reducing the levels of apoptosis and autophagy, improving cell viability, upregulating the expression of Bcl-2 and downregulating the expression levels of Bax and beclin-1. Additionally, curcumin inhibited the expression of BNIP3 and SIRT1 in the H9c2 myocytes subjected to H/R. Therefore, these findings demonstrated the protective effect of curcumin against myocardial H/R injury by reducing the levels of apoptosis and autophagy. Curcumin may offer a potential therapeutic approach for preventing myocardial I/R injury.

\section{Acknowledgements}

The authors would like to thank Dr Zuoquan Xie for their technical assistance. This study was supported by grants 
from the National Natural Science Foundation of China (no. 81102837), the Natural Science Foundation of Zhejiang Province (no. LY13H280004) and the Key Construction Academic Subject (Traditional Chinese Medicine) of Zhejiang Province (no. 2012-XK-A28).

\section{References}

1. Keeley EC, Boura JA and Grines CL: Primary angioplasty versus intravenous thrombolytic therapy for acute myocardial infarction: a quantitative review of 23 randomised trials. Lancet 361: 13-20, 2003.

2. Hausenloy DJ and Yellon DM: Myocardial ischemia-reperfusion injury: A neglected therapeutic target. J Clin Invest 123 92-100, 2013

3. Yellon DM and Hausenloy DJ: Myocardial reperfusion injury. N Engl J Med 357: 1121-1135, 2007.

4. Ong SB and Gustafsson AB: New roles for mitochondria in cell death in the reperfused myocardium. Cardiovasc Res 94: 190-196, 2012.

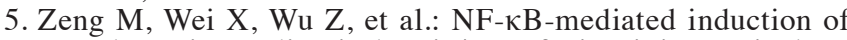
autophagy in cardiac ischemia/reperfusion injury. Biochem Biophys Res Commun 436: 180-185, 2013.

6. Wagner C, Tillack D, Simonis G, Strasser RH and Weinbrenner C: Ischemic post-conditioning reduces infarct size of the in vivo rat heart: role of PI3-K, mTOR, GSK-3beta, and apoptosis. Mol Cell Biochem 339: 135-147, 2010

7. Ma X, Liu H, Foyil SR, Godar RJ, Weinheimer CJ and Diwan A Autophagy is impaired in cardiac ischemia-reperfusion injury. Autophagy 8: 1394-1396, 2012.

8. Yin Y, Guan Y, Duan J, et al: Cardioprotective effect of Danshensu against myocardial ischemia/reperfusion injury and inhibits apoptosis of H9c2 cardiomyocytes via Akt and ERK1/2 phosphorylation. Eur J Pharmacol 699: 219-226, 2013.

9. Hadi NR, Yusif FG, Yousif M and Jaen KK: Both castration and goserelin acetate ameliorate myocardial ischemia reperfusion injury and apoptosis in male rats. ISRN Pharmacol 2014: 206951, 2014.

10. Hadi NR, Al-Amran F, Yousif M and Zamil ST: Antiapoptotic effect of simvastatin ameliorates myocardial ischemia/reperfusion injury. ISRN Pharmacol 2013: 815094, 2013.

11. Levine B and Klionsky DJ: Development by self-digestion: molecular mechanisms and biological functions of autophagy. Dev cell 6: 463-477, 2004.

12. Loos B, Genade S, Ellis B, et al: At the core of survival: autophagy delays the onset of both apoptotic and necrotic cell death in a model of ischemic cell injury. Exp Cell Res 317: 1437-1453, 2011

13. Levine B and Yuan J: Autophagy in cell death: An innocent convict? J Clin Invest 115: 2679-2688, 2005.

14. Matsui Y, Takagi H, Qu X, et al: Distinct roles of autophagy in the heart during ischemia and reperfusion: roles of AMP-activated protein kinase and Beclin 1 in mediating autophagy. Circ Res 100: 914-922, 2007.

15. Kang M, Kim JH, Cho C, et al: Effect of Acori graminei Rhizoma on contractile dysfunction of ischemic and reperfused rat heart Biol Pharm Bull 29: 483-488, 2006.

16. Kang M, Kim JH, Cho C, et al: Anti-ischemic effect of Aurantii Fructus on contractile dysfunction of ischemic and reperfused rat heart. J Ethnopharmacol 111: 584-591, 2007.

17. Abe Y, Hashimoto S and Horie T: Curcumin inhibition of inflammatory cytokine production by human peripheral blood monocytes and alveolar macrophages. Pharmacol Res 39: 41-47, 1999.

18. Dhandapani KM, Mahesh VB and Brann DW: Curcumin suppresses growth and chemoresistance of human glioblastoma cells via AP-1 and NF-kappaB transcription factors. J Neurochem. 102: 522-538, 2007.

19. Kapakos G, Youreva V and Srivastava AK: Cardiovascular protection by curcumin: molecular aspects. Indian J Biochem Biophys 49: 306-315, 2012.

20. Yucel AF, Kanter M, Pergel A, Erboga M and Guzel A: The role of curcumin on intestinal oxidative stress, cell proliferation and apoptosis after ischemia/reperfusion injury in rats. J Mol Histol 42: 579-587, 2011.

21. Shen SQ, Zhang Y, Xiang JJ and Xiong CL: Protective effect of curcumin against liver warm ischemia/reperfusion injury in rat model is associated with regulation of heat shock protein and antioxidant enzymes. World J Gastroenterol 13: 1953-1961, 2007.
22. Awad AS and El-Sharif AA: Curcumin immune-mediated and anti-apoptotic mechanisms protect against renal ischemia/reperfusion and distant organ induced injuries. Int Immunopharmacol 11: 992-996, 2011.

23. Wang L, Li C, Guo H, Kern TS, Huang $\mathrm{K}$ and Zheng L: Curcumin inhibits neuronal and vascular degeneration in retina after ischemia and reperfusion injury. PloS One 6: e23194, 2011.

24. Gonzalez-Salazar A, Molina-Jijon E, Correa F, et al: Curcumin protects from cardiac reperfusion damage by attenuation of oxidant stress and mitochondrial dysfunction. Cardiovasc Toxicol 11: 357-364, 2011.

25. Huang Z, Wang C, Wei L, et al: Resveratrol inhibits EMMPRIN expression via P38 and ERK1/2 pathways in PMA-induced THP-1 cells. Biochem Biophys Res Commun 374: 517-521, 2008.

26. Levine B, Sinha S and Kroemer G: Bcl-2 family members: Dual regulators of apoptosis and autophagy. Autophagy 4: 600-606, 2008.

27. Tanida I, Ueno T and Kominami E: LC3 conjugation system in mammalian autophagy. Int J Biochem Cell Biol 36: 2503-2518, 2004

28. Kroemer G, Galluzzi L, Vandenabeele P, et al: Classification of cell death: Recommendations of the nomenclature committee on cell death. Cell Death Differ 16: 3-11, 2009.

29. Wagener FA, Dekker D, Berden JH, et al: The role of reactive oxygen species in apoptosis of the diabetic kidney. Apoptosis 14: 1451-1458, 2009.

30. Tian Y, Zhang W, Xia D, et al: Postconditioning inhibits myocardial apoptosis during prolonged reperfusion via a JAK2-STAT3-Bcl-2 pathway. J Biomed Sci 18: 53, 2011.

31. Fletcher JI, Meusburger S, Hawkins CJ, et al: Apoptosis is triggered when prosurvival Bcl-2 proteins cannot restrain Bax. Proc Natl Acad Sci USA 105: 18081-18087, 2008.

32. Kim H, Rafiuddin-Shah M, Tu HC, et al: Hierarchical regulation of mitochondrion-dependent apoptosis by BCL-2 subfamilies. Nat Cell Biol 8: 1348-1358, 2006.

33. Takagi H, Matsui Y and Sadoshima J: The role of autophagy in mediating cell survival and death during ischemia and reperfusion in the heart. Antioxid Redox Signal. 9: 1373-1381, 2007.

34. Takagi H, Matsui Y, Hirotani S, et al: AMPK mediates autophagy during myocardial ischemia in vivo. Autophagy 3: 405-407,2007.

35. Fu LL, Cheng Y and Liu B: Beclin-1: autophagic regulator and therapeutic target in cancer. Int J Biochem Cell Biol 45: 921-924, 2013.

36. Zhang J and Ney PA: Role of BNIP3 and NIX in cell death, autophagy, and mitophagy. Cell Death Differ 16: 939-946, 2009.

37. Shin BH, Lim Y, Oh HJ, et al: Pharmacological activation of Sirtl ameliorates polyglutamine-induced toxicity through the regulation of autophagy. PloS One 8: e64953, 2013.

38. Sinha S and Levine B: The autophagy effector Beclin 1: A novel BH3-only protein. Oncogene 27: S137-148, 2008.

39. Przyklenk K, Dong Y, Undyala VV and Whittaker P: Autophagy as a therapeutic target for ischaemia/reperfusion injury? Concepts, controversies, and challenges. Cardiovasc Res 94: 197-205, 2012.

40. Valentim L, Laurence KM, Townsend PA, et al: Urocortin inhibits Beclin1-mediated autophagic cell death in cardiac myocytes exposed to ischaemia/reperfusion injury. J Mol Cell Cardiol 40:846-852, 2006.

41. Marquez RT and Xu L: Bcl-2:Beclin 1 complex: Multiple, mechanisms regulating autophagy/apoptosis toggle switch. Am J Cancer Res 2: 214-221, 2012.

42. Pattingre S, Tassa A, Qu X, et al: Bcl-2 antiapoptotic proteins inhibit Beclin 1-dependent autophagy. Cell 122: 927-939, 2005.

43. Liang XH, Kleeman LK, Jiang HH, et al: Protection against fatal Sindbis virus encephalitis by beclin, a novel Bcl-2-interacting protein. J Virol 72: 8586-8596, 1998.

44. Akar U, Chaves-Reyez A, Barria M, et al: Silencing of Bcl-2 expression by small interfering RNA induces autophagic cell death in MCF-7 breast cancer cells. Autophagy 4: 669-679, 2008.

45. Hamacher-Brady A, Brady NR, Logue SE, et al: Response to myocardial ischemia/reperfusion injury involves Bnip3 and autophagy. Cell Death Differ 14: 146-157, 2007.

46. Bellot G, Garcia-Medina R, Gounon P, et al: Hypoxia-induced autophagy is mediated through hypoxia-inducible factor induction of BNIP3 and BNIP3L via their BH3 domains. Mol Cell Biol 29: 2570-2581, 2009.

47. Yasuda M, Theodorakis P, Subramanian T and Chinnadurai G: Adenovirus E1B-19K/BCL-2 interacting protein BNIP3 contains a $\mathrm{BH} 3$ domain and a mitochondrial targeting sequence. J Biol Chem 273: 12415-12421, 1998 . 
48. Chen G, Ray R, Dubik D, et al: The E1B 19K/Bcl-2-binding protein Nip3 is a dimeric mitochondrial protein that activates apoptosis. J Exp Med 186: 1975-1983, 1997.

49. Hariharan N, Maejima Y, Nakae J, Paik J, Depinho RA and Sadoshima J: Deacetylation of FoxO by Sirt1 plays an essential role in mediating starvation-induced autophagy in cardiac myocytes. Circ res 107: 1470-1482, 2010.

50. Lee IH, Cao L, Mostoslavsky R, et al: A role for the NAD-dependent deacetylase Sirtl in the regulation of autophagy. Proc Natl Acad Sci USA 105: 3374-3379, 2008.
51. Ou X, Lee MR, Huang X, Messina-Graham S and Brox meyer HE: SIRT1 positively regulates autophagy and mitochondria function in embryonic stem cells under oxidative stress. Stem Cells 32: 1183-1194, 2014.

52. Lee Y, Lee HY, Hanna RA and Gustafsson AB: Mitochondria autophagy by Bnip3 involves Drp1-mediated mitochondrial fission and recruitment of Parkin in cardiac myocytes. Am J Physiol Heart Circ Physiol 301: H1924-1931, 2011. 